of the smooth plains, wrinkle ridges and lobate scarps observed. Unquestionably to some, the plains areas appear filled as a result of internal activity. But yet how is it possible for younger craters to be floored with plains-like looking material which the cratering process should have obviously disturbed? The view was also expressed that the intercrater plains represent a process contemporary with the cratering and occur as a result of impact melting. The debate is reminiscent of the lunar questions in the mid-1960s following the early lunar orbiter photographs but prior to the Apollo landings.

It is readily admitted that it is not yet possible to consider development of a single model of evolution of this planet. In addition to its anomalously high average density, we now know its surface to be very Moon-like and yet with its modest magnetic field its interior must be more Earth-like. Where Mercury fits in the grand scheme of the origin of the terrestrial planets is not certain. It can reasonably be expected that future studies of these results and comparisons with the Moon and Mars (with the USA's Viking 76 landers) will shed light on this fundamental question.

\section{Human cytogenetic registries}

from a Correspondent

THE astonishing success of human cytogeneticists in communicating their discoveries in a standardised and comprehensible way is due to their early recognition of the need for a standard system of nomenclature. To this end, participants at four international meetings have made recommendations and devised systems of nomenclature, each of which has extended and modified the previous one and thus successfully kept pace with rapid technical advances. The meetings were arranged on an ad hoc basis and had no authority save their broadly based international representation and the excellent reports which resulted. Their recommendations have been followed by virtually all workers in human cytogenetics.

The question of an international registry of abnormal human karyotypes was first raised in 1966 because many people felt that unpublished data on individuals with abnormal chromosomes (and all laboratories of human cytogenetics have such data) could, if collected and pooled, be used in some worthwhile way. The topic was first considered in depth on April 7 and 8 of this year when a standing committee on human cytogenetics, set up after the 1971 international meeting held in Paris, sponsored a workshop in Edinburgh on human cytogenetic registries. The meeting was attended by nine individuals who run such registries, by consultants and by members of the standing committee.

The workshop first considered cytogenetic registries generally. It was agreed that it was absolutely essential that the aims and goals of a cytogenetic registry be clearly defined and that the output of useful data from such a registry was dependent upon, and limited by, the methods of ascertainment employed in the registry and by the quality and uniformity of the information within it. Subsequent to discussion of the methods of intake into a registry, the question of patient confidentiality, the rigorous quality control needed for data in the registry and the necessity for the continued surveillance of patients for certain types of registries, the participants turned their attention to the question of an international registry of abnormal human karyotypes. After considering a variety of problems to which cytogenetic registries might address themselves, the participants decided that their solution would not be helped by an international registry.

Therefore, with one dissension (Dr D. S. Borgaonkar: see Nature, 253, 591; 1975) they recommended that an international cytogenetic registry should not be set up at this time and that if, at some future date, an international registry should be considered of value, it should be established only after careful planning and under the guidance of an international advisory committee. They also recommended that the establishment of regional and national cytogenetic registries should be encouraged, as should cooperation between them and existing registries. They further advised, again with one dissension, that an international advisory committee on cytogenetic registries should be set up by the standing committee of the Paris conference and, lastly, that the standing committee should advise international organisations and appropriate national agencies of these developments and solicit their cooperation and support.

The workshop report was accepted by the standing committee who will publish it later this year as part of a supplement to the Paris Nomenclature. In the light of the recommendations the standing committee has already established an advisory committee on human cytogenetic registries under the chairmanship of Dr James R. Miller, Department of Medical Genetics, University of British Columbia, Vancouver.

At a time when few regional or national registries have yet shown their worth, it seems that the Edinburgh workshop report reflects a very sensible decision not to support at present the establishment of an international cytogenetics registry which would, in all probability, turn into a cumbersome, expensive and unproductive way of disposing of unpublished data.

\section{Label triangulation becomes feasible}

from a Correspondent

A Brookhaven Symposium on neu-
tron scattering in biological research
was held on June 2-6 at the Brook-
haven National Laboratory, New
York.

INVESTIGATING the topography of ribosomal proteins by the chemical methods discussed recently in Nature (254, 555; 1975) can only lead to a qualitative model of a ribosome. The standard methods for structure determination are diffraction methods. At the symposium two groups showed independently that the application of the label triangulation method' to ribosomes has become an experimental reality. This method is in principle capable of giving the complete quaternary structure of the 54 proteins in a ribosome in a quantitative and unambiguous way. It is based on low-angle scattering in solution and was first proposed for $\mathrm{X}$ rays (Hoppe, Israel J. Chem., 10, 321; 1972) and shortly thereafter for neutrons (Engelman and Moore, Proc. natn. Acad. Sci. U.S.A., 69, 1997; 1972).

Scattering in solutions can be treated like gas diffraction. Using the corresponding theory it can be shown that the linear combination of four scattering curves $\mathrm{u}=\mathrm{a}+\mathrm{b}-\mathrm{c}-\mathrm{d}$ (scattering of solutions of equal amounts of $a$, the molecular complex to be studied; $b$, the molecular complex labelled with two additional scatterers $\mathrm{A}$ and $\mathrm{B}$; $\mathrm{c}$, the molecular complex labelled with $A$ only; d, the molecular complex labelled with B) corresponds to a damped sine curve with a wavelength inversely proportional to the distance $\mathrm{AB}$ (see also Kratky et al., Mh. Chem., 76, 281; $1946 ; 78,295 ; 1948)$. A systematic labelling is possible by disaggregation of the ribosome (respectively of the $30 \mathrm{~S}$ or $50 \mathrm{~S}$ subunit) and by reaggregation, replacing unlabelled proteins by labelled proteins. In the case of $\mathrm{X}$ rays labelling can be done by introducing heavy atoms; in case of neutrons by replacing deuterium (in a completely deuterated subunit) with hydrogen. A triangulaiion of the distances leads to the quaternary structure. Approximately 200 distances can be measured in the $30 \mathrm{~S}$ subunit and 500 in the $50 \mathrm{~S}$ subunit. 